\title{
Prevención del SIDA y Relación entre Géneros: Poder y Cambio
}

\author{
AIIDS Prevention and the Relations \\ hip between the Sexes: Power and Change \\ Mariela Carmona Sepúlveda* \\ Universidad Santo Tomás de Chile
}

(Recepción: Abril 2004 - Aceptación: Junio 2004)

\begin{abstract}
Este documento pretende ser una reflexión sobre la adopción (o más bien sobre la no adopción) de prácticas o conductas preventivas en parejas estables ${ }^{1}$ chilenas frente a la epidemia del SIDA. Dichas prácticas implican necesariamente un proceso de negociación ${ }^{2}$ de pareja en todo lo referente a la sexualidad y al uso del preservativo masculino como principal método de prevención. La construcción de una reflexión de este tipo implica necesariamente el abordar la relación hombre-mujer considerando la diferencia de géneros como una construcción social, pero también como una relación de poder, plasmada en las representaciones o significaciones sociales en torno a la sexualidad y a la enfermedad. O en términos de Castoriadis, es necesario tratar de entender el imaginario social de hombres y mujeres de un segmento particular de la sociedad chilena. Para esta reflexión, tomaremos prestados algunos conceptos mencionados por Norma Takeuti con ocasión de su intervención en el curso «Teorías del Poder» ${ }^{3}$, especialmente a partir de los trabajos de Castoriadis, Foucault y Bourdieu 4 .

Palabras clave: Género, poder, prevención.
\end{abstract}

This document reflects on stable Chilean couples' use of (or rather their failure to use) preventive practices or behaviors in response to the AIDS epidemic. These practices necessarily imply a negotiation process on the part of the couple towards sexuality overall and the man's use of the condom as the primary method of prevention. As we delve into this reflection, it is important to consider the gender difference in a relationship between a man and a woman as a social construction, but we must also see it as a power relationship that is manifested in the social representation or significance of sexuality and the disease. Or, as Castoriadis puts it, we need to attempt to understand the social imaginary of the men and woman of a particular segment of Chilean society. In our study, we have borrowed some concepts mentioned by Norma Takeuti in the course "Theories on Power" based particularly on the works of Castoriadis, Foucault, and Bourdieu.

Key words: Gender, power, prevention.

\section{La prevención del SIDA: un proceso de negociación en la pareja}

¿De dónde surge el interés por este tema? A partir de la observación de la implementación de algunos programas de prevención del SIDA,

* Psicóloga, Licenciada en Psicología, Pontificia Universidad Católica de Chile.

Magíster en Sociología, Universidad de París VII. Doctora (c) en Ciencias Sociales, Universidad de París V.

Correspondencia: Escuela de Psicología, Universidad Santo Tomás. Avda. Ejército Libertador 146, Santiago-Chile. (56-2) 6324888.

E-mail: renaico@hotmail.com

1 Pareja estable no en el sentido de exclusividad sino que de permanencia en el tiempo.

2 Coria (1994) define la negociación como «un proceso interactivo, en el cual las personas implicadas tienen intereses distintos y desarrollan acuerdos con el fin de obtener aquello que ellas desean de la relación. La negociación es un proceso que puede ser realizado en dominios tan diferentes como el personal, afectivo o sexual». y de la percepción (o intuición) de que ellos no serían siempre eficaces ya que se basarían sólo en la entrega de conocimientos técnicos sobre la enfermedad y sus formas de contagio, o estarían enfocados sólo hacia una «toma de conciencia» de la propia sexualidad, de la condición de la mujer con respecto a la relación de dominación entre géneros y de los peligros de la enfermedad. Así, algunos de estos programas y de los programas generales sobre salud sexual y reproductiva promoverían «el empoderamiento $)^{5}$ de las mujeres, adolescentes y niñas en todos los ámbitos sociales, por medio de su concientización de género, y del

3 DEA «Sociologie du Pouvoir: politiques, institutions et sociétés», Laboratoire de Changement Social, Université Paris VII. París, 2000-2001.

4 Traducciones libres de los textos originales en francés, realizados por la autora del artículo.

5 Concepto anglosajón que significa «reforzamiento del poder») (Vidal, 2000). 
ejercicio y la defensa de sus derechos humanos y ciudadanos ${ }^{6}$.

¿Es esto suficiente? ¿Posibilitan siempre estas acciones un cambio en las conductas de riesgo y en las prácticas de prevención tales como la negociación de las condiciones en que se desarrolla la vida sexual de la pareja y la adopción del uso del preservativo masculino? En las evaluaciones realizadas al final de cada intervención de este tipo, parece siempre haber un buen nivel de aprendizaje teórico, pero ¿tiene verdaderamente este discurso su repercusión en la práctica? ¿Son los discursos sobre la dominación más «avanzados» que las prácticas mismas? ¿Llegan efectivamente hombres y mujeres realmente a negociar sobre las condiciones de su sexualidad?

Si la respuesta a esta última pregunta es negativa, y si suponemos que el temor al contagio y a la muerte deberían ser condiciones suficientes para la implementación de conductas de prevención, ¿cómo explicar la aparente «irracionalidad» de la no adopción de estas conductas? ¿Qué es lo que dificultaría o impediría la posibilidad de negociación en la pareja? ¿Se llega efectivamente a negociar o ni siquiera este punto es alcanzado?

Lo anterior nos lleva a reflexionar sobre el hecho de que los aprendizajes teóricos sobre la negociación sexual son necesarios pero insuficientes, ya que existiría otro tipo de obstáculos más bien ligados a la adhesión inconsciente a los modelos de comportamiento sexual y a las relaciones de poder entre géneros.

Esta distinción entre conciencia e inconsciencia nos lleva a pensar en la polémica mencionada por Takeuti ${ }^{7}$ sobre la noción marxista de ideología como «falsa conciencia» o la idea de que «algo ha sido invertido de la conciencia humana» y que sólo bastaría con invertir la in-

6 Lo anterior conforma uno de los ítems de las «Recomendaciones para la acción: salud y derechos sexuales y reproductivos en América Latina y el Caribe», documento que recoge algunos de los puntos que forman parte de los acuerdos tomados por los países de la región en las últimas conferencias y foros mundiales (Beijing, 1995; El Cairo+4, 1999) y que resume algunas propuestas surgidas de evaluaciones recientes de la implementación de dichos acuerdos (Agenda de salud : Isis Internacional, $\left.\mathrm{n}^{\circ} 10,1998\right)$.

7 En su texto «Imaginário social 'mortífero': A questão da delinqüência juvenil no Brasil», en el contexto de su intervención en el DEA Sociologie du Pouvoir, Laboratoire de Changement Social, Paris 7, noviembre 2000. versión realizada (podríamos decir hacer consciente 1o inconsciente en términos psicoanalíticos). Es decir, se sugiere la idea de un grupo dominado por otro e inconsciente de su propia realidad o forjándose representaciones erróneas sobre su condición. Sería fácil trasladar estas ideas a la dominación de hombres sobre mujeres, y sugerir que las mujeres no llegan a negociar porque son oprimidas sexualmente por sus parejas, opresión de la cual ellas muchas veces no son conscientes. Es posible pensar así que la tarea de las instituciones que se encargan de la promoción de sus derechos es develar estas injusticias y promover en las mujeres su capacidad de decisión y de autonomía frente a este «enemigo principal» ${ }^{8}$.

Creemos que esta problemática reviste una complejidad mucho mayor, entre otras cosas, porque no es posible pensar en las mujeres como no conscientes de esta dominación (dominación que por lo demás tampoco se pretende negar, existen numerosos ejemplos de ello en distintos ámbitos tanto en el mundo privado como en el mundo público); muchas veces ellas lo saben, pero también participan en su reproducción a través de la adhesión inconsciente a modelos de relación que determinan sus prácticas. Podríamos decir entonces, reformulando una vez más la fórmula marxiana de "ellos no saben de eso, pero lo hacen», por «ellas pueden llegar a saber lo que hacen, pero aun así lo hacen». Esto no implica una alusión al concepto de razón cínica de Sloterdijk $(1987)^{9}$, el que habla de la plena conciencia de los dominados de un interés oculto detrás de una dominación, pero aun así la no renunciación a ella, sino más bien nuevamente al hecho de que no basta con la toma de conciencia de la relación de poder hombremujer para salir de ésta.

Para Bourdieu (1998), «el efecto de la dominación simbólica (sea ésta de etnias, de género, de culturas, de lenguas, etc.) no se ejerce dentro de la lógica pura de conciencias cognoscentes, sino que más bien a través de esquemas de percepción, de apreciación y de acción constitutivos de los habitus, fundando, más allá de las decisiones de la conciencia y del control de la voluntad, una relación de conocimiento

8 Término utilizado por C. Delphy en el contexto del feminismo radical, para referirse al patriarcado como la principal forma de dominación.

9 Sloterdijjk, P. 1987. Crítica de la razón cínica. París: Bourgeois. En Op. cit. 7. 
profundamente oscura a ellas mismas» (p. 43). Y agrega: «Las pasiones del habitus del dominado no son aquellas que pueden ser suspendidas por un simple esfuerzo de la voluntad, fundado sobre una toma de conciencia liberadora. Si el creer que la violencia simbólica puede vencerse sólo con las armas de la conciencia y de la voluntad es completamente ilusorio, es porque los efectos y las condiciones de su eficacia están doblemente inscritos en lo más íntimo de los cuerpos bajo la forma de disposición» (p. 45).

¿Cuáles serían entonces estos mecanismos inconscientes de adhesión a los modelos de relación, especialmente en lo que dice relación con la sexualidad? Antes de abordar esta pregunta, queremos destacar la importancia de trabajar este tema doblemente, es decir, desde la perspectiva femenina pero también desde la perspectiva masculina. Es decir, no sólo dirigir la investigación sobre el género o sobre la prevención de SIDA hacia las mujeres. Fácil es caer en esta trampa, si adoptamos una postura de dominador-ostentor de poder (consciente)/dominada-sin poder alguno (inconsciente). Como acabamos de decir, las cosas parecen ser mucho más complejas que eso, ya que tanto hombres como mujeres adherimos sin darnos cuenta a formas de visualizar el mundo que determinan nuestras prácticas en él. Como señala Takeuti ${ }^{10}$, ya no es posible sostener la idea de una realidad «falseada» por un grupo social que procura asegurar el poder de la dominación sobre otro.

Considerar ambos sexos evita caer en la noción recurrente de «vulnerabilidad» de las mujeres, lo que lleva una vez más a pensar de acuerdo a los polos dominante-dominado y a reafirmar esta dicotomía como realidad única (discutiremos esta concepción del poder más adelante, sobre todo a partir de las teorías de Foucault). Por otro lado, esto plantea también un problema desde el punto de vista de la «distribución» de la responsabilidad: la mujer sería la responsable de promover la negociación del uso del preservativo y el fracaso se debería a que ésta no ha desarrollado suficiente autonomía para decidir sobre su propio cuerpo. Pero ¿dónde queda la responsabilidad de su pareja en esta negociación?

Vidal (2000) en sus trabajos sobre África plantea algo semejante al señalar la presencia de fuertes estereotipos en los trabajos sobre el

10 Op. cit. 7.
SIDA: «Por su parte, la asociación 'SIDA y mujeres', pone en primer plano prácticas masivas de prostitución, 'culpables' de la epidemia y, en el polo opuesto, situaciones de gran 'vulnerabilidad' de las mujeres, imposibilitadas de negociar con sus parejas la utilización del preservativo. Por último, los discursos sobre las 'mujeres y África', retoman sin cesar las imágenes de sumisión de las mujeres a la dominación masculina, expresiones de 'relaciones de género' tradicionalmente y definitivamente desiguales, las que deben ser enfrentadas a través de estrategias dirigidas a reforzar el 'poder' y la capacidad de decisión de las mujeres» (p. 2).

Este mismo autor propone la idea del SIDA como revelador y transformador social, diciendo que esta concepción no es en absoluto novedosa: «la forma en que las mujeres africanas enfrentan el SIDA revela la naturaleza de múltiples situaciones vividas por ellas mismas (relación con el hombre, con el deseo infantil, con otras generaciones, con la enfermedad, con los cuidados, etc.) y acelera su evolución» ( $p$. 11). Tomaremos esta idea para continuar nuestra reflexión.

¿Cómo poder comprender la relación entre hombres y mujeres, que el SIDA vendría a revelar? La amenaza del SIDA, ¿viene a acelerar o más bien a enlentecer los procesos de transformación social de la relación entre géneros?

El SIDA como revelador: la dominación masculina y las significaciones imaginarias sociales

Es posible decir que el SIDA contribuiría a revelar la asimetría del poder en la relación entre hombres y mujeres con respecto a su sexualidad, producto de un determinado modo de representarse la relación entre ambos géneros. Nos parece que más que revelar esta situación de dominación, presente en la mayor parte de las sociedades occidentales, las fuertes contradicciones que genera la necesidad de prevención de esta enfermedad permiten profundizar en la cuestión de los mecanismos que perpetúan este modo de representación, aun frente a la «irracionalidad» de la amenaza de la propia desintegración o la de la pareja.

Bourdieu reflexiona sobre estos poderosos mecanismos inconscientes, y señala: «la representación androcéntrica de la reproducción biológica y de la reproducción social se encuentra investida de la objetividad de un sentido común, entendido como un consenso práctico, de la doxa, sobre el sentido de los comportamientos. 
Y las mismas mujeres aplican a toda realidad, particularmente a las relaciones de poder en las que ellas se encuentran encerradas, esquemas de pensamiento que son el producto de la incorporación de estas relaciones de poder, expresadas en las oposiciones fundadoras del orden simbólico. Esto tiene como consecuencia que sus actos son, por este mismo camino, actos de reconocimiento práctico, de adhesión dóxica, creencia que no debe ser pensada sino que ser afirmada por sí misma, «haciendo», de alguna manera, la violencia simbólica» (Bourdieu, 1998, p. 39).

La violencia simbólica «se instituye por intermedio de la adhesión que el dominado no puede no acordar al dominador (por lo tanto a la dominación), cuando no dispone, para pensarlo y para pensarse o, mejor, para pensar la relación, de otros instrumentos de conocimiento que los que tienen en común, y que, tomando la forma de la relación de dominación, hacen aparecer esta relación como natural» (Bourdieu, 1998, p. 41).

Estas ideas nos parecen interesantes ya que insinuarían la existencia de «instrumentos de conocimiento» en común que determinarían la adhesión de ambos sexos a la dominación como modo de relación y a su institucionalización bajo la forma de violencia simbólica, lo que nos evita volver a caer en la trampa de pensar reductivamente en un género masculino ejerciendo una dominación voluntaria sobre un género femenino que no se da cuenta de ello.

Otros autores se han interrogado sobre lo que podríamos llamar esta «naturalización» de la relación de dominación como institución de una sociedad determinada. Aunque no se refiere específicamente a la relación de dominación entre géneros, Castoriadis, en «La institución imaginaria de la sociedad» (1975), hablando sobre las instituciones en general, reflexiona sobre la manera como éstas llegan a autonomizarse. Él señala: «La alienación, es la autonomización y el dominio del momento imaginario dentro de la institución. Esta autonomización de la institución se expresa y se encarna en la materialidad de la vida social, pero supone siempre que la sociedad se relaciona con sus instituciones bajo el modo del imaginario, y que ella no reconoce en este imaginario de las instituciones su propio producto» (Castoriadis, 1975, p. 198).
Nos interesamos especialmente en los planteamientos de este autor por dos razones principales. La primera, porque éste nos propone, por una parte, la existencia de un mundo de significaciones imaginarias sociales (imaginario social) que determinaría el modo de hacer y de representarse/decir de una sociedad. Pero ellos nos permiten también pensar en una «adhesión inconsciente» de los individuos a sus instituciones: él reflexiona sobre la autonomización de las instituciones dentro de una sociedad y sobre la alienación como modalidad de acercamiento a ellas. Las creaciones imaginarias (las significaciones imaginarias sociales, por ejemplo: la reificación, la consideración de una categoría de hombres superiores a otros) no necesitan ser explicitadas en los conceptos o en las representaciones para existir, ellas actúan en la práctica y en el hacer de la sociedad, y tienen un sentido organizador del comportamiento humano y de las relaciones sociales, independientemente de su existencia para la «conciencia» de esa sociedad (Castoriadis, 1975).

¿Podríamos entender a partir de lo anterior la fuerza de los modelos de relación de pareja en cuanto a la sexualidad y a la dificultad de negociar conductas preventivas frente a la epidemia del SIDA? ¿Cuáles serían en este caso las significaciones imaginarias sociales que dan sentido al mantenimiento de representaciones y prácticas que ponen a las personas en riesgo de contaminación? ¿Podemos acceder a ellas de otra forma que a través de la interpretación de lo que los individuos hacen y se representan? Representaciones como, por ejemplo, la necesidad de satisfacción sexual «más fuerte» en hombres que mujeres, lo que permite la infidelidad masculina, su aceptación y a veces incluso su justificación por parte de las mujeres, y el rechazo al uso del preservativo, ya que éste disminuiría el placer sexual del hombre y además sería un modo de promover la fantasía de la infidelidad femenina, mucho menos aceptada. O, relacionado con lo anterior, la predominancia del «erotismo» en la sexualidad masculina y de la «afectividad» en la sexualidad femenina (Rivera, Rodó, Sharim \& Silva, 1995).

Adentrarse en el imaginario social se plantea como un desafío ineludible. Por el momento, podríamos sólo plantearnos algunas preguntas: ¿son consideradas las mujeres como «bie- 
nes simbólicos» a capitalizar, en términos de Bourdieu, a propósito de la sociedad cabila? ${ }^{11}$ ¿Se refiere el poder masculino al dominio del placer femenino? O como lo plantea De Barbieri (1992), ¿se basa este poder en el control de la capacidad reproductiva frente a la necesidad de trascendencia?

Es posible preguntarse también sobre las significaciones imaginarias que determinarían el modo de representarse el SIDA como enfermedad, y el rechazo del preservativo en su función preventiva: ¿por qué estamos tan lejos de adoptar comportamientos «racionales» frente a la enfermedad y a la muerte? «¿Por qué y cómo este imaginario, una vez instalado, tiene consecuencias propias, que van más allá de sus motivos funcionales e incluso los contradicen, las que permanecen después de las circunstancias que las hicieron nacer, y que finalmente muestran un factor autonomizado de la vida social?» (Castoriadis, 1975, p. 193).

En segundo lugar, nos interesamos en las tesis de este autor porque también nos ayudan a sobrepasar la dicotomía de la relación de poder dominante-dominado: «La alineación aparece como una modalidad de relación con la institución (...) La superación de la alienación presupone la eliminación de la dominación de toda clase particular, pero va más allá de este aspecto (...) en una sociedad alienada la misma clase dominante está en una situación de alienación: sus instituciones no son exteriores a ella misma, ellas están autonomizadas a instancias de la sociedad (...) Es necesario comprender qué es lo que se autonomiza, por qué y cómo» (Castoriadis, 1975, p. 171).

Las elecciones que una sociedad hace para poder responder a sus necesidades se sustentan en una serie de significaciones imaginarias que valorizan y desvalorizan, estructuran y jerarquizan un conjunto cruzado de objetos y

11 El principio de la inferioridad y de la exclusión de la mujer (...) no es otra cosa que la asimetría fundamental del sujeto y el objeto, del agente y del instrumento, que se instaura entre el hombre y la mujer en el terreno de los intercambios simbólicos, de las relaciones de producción del capital simbólico, en el cual el dispositivo central es el mercado matrimonial, y que están a la base de todo el orden social: las mujeres no pueden aparecer ahí de otra forma que en tanto objetos o, mejor, en tanto símbolos cuyo sentido está constituido fuera de ellas y cuya función es contribuir a la perpetuación o al aumento del capital simbólico detentado por los hombres (Bourdieu, 1998, p. 49). de carencias correspondientes, sobre lo cual puede leerse también esa cosa tan incierta como incontestable que es la orientación de una sociedad (Castoriadis, 1975, p. 226). Los oprimidos se mantienen de mil formas tributarios del imaginario que ellos combaten en alguna de sus manifestaciones, y muy seguido lo que ellos buscan no es más que una permutación de los roles sobre el mismo escenario; muy luego, sin embargo, la clase oprimida responde negando en bloque el imaginario social que la oprime ${ }^{12}$ (Castoriadis, 1975, p. 235).

Si consideramos todo lo anterior, ¿podríamos tratar de entender la fuerza de la dominación masculina como proveniente de un sistema de significaciones sociales imaginarias que permite la permanencia de una determinada forma de relación entre ambos géneros, más que como el ejercicio consciente de un determinado grupo sobre otro? Castoriadis (1990), en «Poder, política, autonomía» ${ }^{13}$, señala que: «previo a todo poder explícito o dominación, la institución de la sociedad ejerce un infrapoder radical (manifestación y dimensión del poder instituyente del imaginario radical), que no es localizable. No es ciertamente el de un individuo o incluso de una instancia designable (...) $\mathrm{El}$ infrapoder, el poder instituyente, es a la vez aquel del imaginario instituyente, de la sociedad instituida (...) es entonces el poder del dominio social-histórico por él mismo, el poder d'outis, de Nadie (Castoriadis, 1990, p.144).

En esta misma línea, Foucault, en «La Voluntad de Saber» (1976), critica la concepción jurídico-política del poder, que concibe el poder como ejerciéndose de la misma manera a todo nivel: «Desde lo alto hacia lo bajo, en las decisiones globales como en las intervenciones capilares, sean los que sean los aparatos o las instituciones sobre las cuales se apoya, el poder actuaría de manera uniforme y masiva (...) desde el Estado hacia la familia, desde el príncipe al padre, desde el tribunal al más mínimo de los castigos cotidianos, desde las instancias de dominación social a las estructuras constitutivas del sujeto, encontraríamos, solamente a diferente escala, una forma general del poder» (p. 111).

12 Cursivas agregadas.

13 En «Les carrefours du labyrinthe 3: Le monde morcelé».. 


\section{CARMONA}

Podríamos pensar entonces que no es posible tomar el poder masculino como un poder predeterminado de una vez por todas. Esta concepción vertical del poder implica que «todas las formas de dominación, de sumisión, de sometimiento, se llevarían finalmente al efecto de obediencia» (Foucault, 1976, p. 113). ¿Es éste siempre el caso en la relación de pareja? ¿Qué repercusiones tiene esto en relación al tema que nos interesa, es decir, la prevención del sida? Pensamos que es esta la visión que predomina en la creación y en la implementación de los programas de prevención basados en la necesidad de «autonomizarse» del poder masculino y de fortalecer el propio poder de la mujer. Por lo demás, un objetivo de este tipo quedaría fijado en la misma concepción de poder desde arriba hacia abajo. Esta visión «no se adecua ciertamente a la manera en la que el poder se ejerce; pero ella es el código según el cual éste se presenta y prescribe que lo pensemos» (Foucault, 1976, p. 116).

Cuando de trata de la utilización del preservativo, ¿es tan simple como promover la capacidad de negociación en la mujer? Si esta negociación no llega a ser posible, ¿basta con decir que esto es resultado de la obediencia femenina ante el poder masculino? Quizás esto sería simplificar demasiado el problema. Por un lado, porque no sólo estamos hablando de relación de dominación entre dos categorías sociales, sino que porque estamos dentro de una relación personal entre dos individuos que forman una pareja, con todo lo que esto implica desde el punto de vista psicológico (afectividad, identidad, necesidad del otro, etc.). ¿Cuál sería el papel del «amor» en esta relación de poder? ¿De qué manera se relaciona éste con las determinaciones sociales? Como plantea Sonia Dayan-Herzburn ${ }^{14}$, sería entonces más adecuado pensar en el poder como un juego con fuerzas en tensión constante, que también se juega en la relación, y no desde arriba y de una vez por todas. De esta forma, es necesario interrogar el poder no sólo desde su componente político, no sólo como poder de un grupo sobre otro, sino que más bien sobre la multiplicidad de fuerzas. Esto incluye por supuesto el problema de la dominación de géneros pero de una manera más compleja.

14 Notas del seminario: «Relaciones de género en el Maghreb y Machreb» EHESS, 2000-2001.
Foucault (1976) escribe: «por poder, me parece que es necesario comprender primero la multiplicidad de relaciones de fuerza que son inmanentes al dominio en el cual ellas se ejercen, y son constitutivas de su organización; el juego que por medio de luchas y de enfrentamientos incesantes las transforma, las refuerza, las invierte» (p. 122). Y más aún: «el poder está en todas partes; no es que él englobe todo, sino que él viene de todas partes (...) El poder no es una institución, y no es una estructura, no es una cierta potencia de la cual algunos estarían dotados: es el nombre que se le presta a una situación estratégica compleja en una sociedad dada» (p. 123).

Ahí donde hay poder, hay resistencia, y sin embargo ésta no se halla jamás en posición de exterioridad en relación al poder (Foucault, 1976). Pagès, de Gaulejac y Bonetti (1987) señalan con respecto al poder en las organizaciones que se trata, ante todo, de una concepción donde el poder es producido, investido, actualizado y utilizado por todos los diversos escalones jerárquicos. No se trata de un poder «trascendental», sino más bien, «inmanente» a los propios individuos que son producidos por el poder, al mismo tiempo que ellos mismos contribuyen a crearlo ${ }^{15}$.

¿Cómo contribuyen hombres y mujeres a crear esta «dominación masculina»? ¿Cómo podríamos pensar esta posible "resistencia» por parte de la mujer en la relación de pareja? ¿En qué otros ámbitos la mujer ejercería a su vez el poder? ¿Tiene poder en otros dominios pero no con respecto a la sexualidad? ¿Sería esto una forma de resistencia? ¿Habría algún tipo de «beneficio secundario» que haría que la mujer (consciente o inconscientemente) ceda en un dominio para obtener más poder en otro? ¿Cómo integrar esto en relación con formas extremas de poder que sufren algunas mujeres como historias de abuso sexual y de violencia intrafamiliar? Si pensamos el problema del poder como una red de relaciones de poder, es al mismo tiempo necesario considerar otras formas de dominación, que no se limitan pero que cruzan esta relación entre géneros, en un país en vías de desarrollo, es-

15 Pagès, M., Gaulejac, V. de, Bonetti, M., Descendre, D. 1979. O poder das organizações. A dominação das multinacionais sobre os individuos. São Paulo: Atlas, 1987. En Op. cit.7. 
pecialmente en sectores sociales que a pesar de lo anterior aún viven en condiciones precarias: las enormes diferencias de poder entre clases sociales, entre etnias diferentes, el acceso desigual a la información, sobre todo en lo que se relaciona con educación y prevención en salud. ¿Cómo integrar todo esto en un país donde por varios años el Poder (aquí escrito con mayúscula) llegó a su estado más absoluto, intentando sofocar toda posibilidad de resistencia?

Lo que nos parece lo más importante de extraer de esta reflexión es nuevamente la idea de que ya no es posible pensar la relación entre hombres y mujeres como una relación en que un grupo domina voluntariamente a otro. Evidentemente, no se trata de descartar la «dominación masculina», no pensamos negar una realidad observable en distintos niveles de la sociedad. Se trata más bien de entender que la permanencia de este modelo de relación de poder entre hombres y mujeres depende de mecanismos de adhesión que no tienen siempre que ver con la conciencia ni con la voluntad de los actores involucrados, y que por lo mismo, todos, tanto hombres como mujeres, contribuimos a su reproducción.

Sin pretender forzar articulaciones teóricas, pensamos, por un lado, en las ideas de Castoriadis: lo social histórico es lo colectivo anónimo, lo humano interpersonal. Existirían, por un lado, estructuras, instituciones, obras 'materializadas' y, por otro, lo que estructura, instituye, materializa. Es la unión y la tensión de la sociedad instituyente (de la historia que se realiza) y de la sociedad instituida (de la historia realizada) ${ }^{16}$. Pensamos también en Foucault (1976) cuando éste señala: «Ningún foco local, ningún esquema de transformación podría funcionar si, por una serie de encadenamientos sucesivos, éste no se inscribiera a fin de cuentas dentro de una estrategia de conjunto (...) es necesario pensar más bien en el doble condicionamiento de una estrategia por la especificidad de tácticas posibles, y de las tácticas por el envoltorio estratégico que las hace funcionar» (p. 132).

16 En Op. Cit. 7.
El SIDA: ¿factor acelerador o retardador de transformaciones sociales? Cambios en los modelos de sexualidad, sociedad instituida $y$ sociedad instituyente

Si pensamos que las significaciones imaginarias sociales actuarían como organizadores de sentido de las prácticas humanas en una época y en una sociedad determinada, estableciendo las líneas de demarcación entre lo lícito y lo ilícito, entre lo permitido y lo prohibido, entre lo representable y lo no representable, podemos decir que éstas consolidarían entonces las condiciones reproductivas del poder instituido o sociedad instituida.

Pero este imaginario social no está dado en forma definitiva, siempre es posible asistir a la emergencia de nuevos organizadores de sentido, que transforman los ya existentes y proponen nuevos sistemas de significaciones. Según Castoriadis (1975): «Aquello que, dentro de lo social-histórico, es posición, creación, hacer ser, lo llamamos imaginario social en el sentido último del término, o sociedad instituyente. Aquello que, dentro de la psyqué/soma es posición, creación, hacer ser para la psyqué/soma, lo llamamos imaginación radical (...) El imaginario social o la sociedad instituyente está dentro y es para la posición-creación de significaciones imaginarias sociales y de la institución como «presentificación» de esas significaciones, y de sus significaciones como instituidas. El imaginario radical está dentro y es para la posición-creación de figuras como presentificación de sentido y de sentido siempre figurado-representado (p. 533).

La sociedad instituida está continuamente siendo trabajada por la sociedad instituyente, bajo el imaginario social establecido fluye siempre el imaginario radical: existe imaginario instituyente (Castoriadis, 1990).

En el área de la sexualidad podemos observar actualmente importantes transformaciones en los modelos instituidos, especialmente en lo que tiene que ver con la definición de lo masculino y de lo femenino y con las relaciones de poder entre géneros. En este sentido, es posible reconocer especialmente durante los años sesenta, un hito importante de transformación y de emergencia de nuevos significados. Hasta este período se podía identificar nítidamente la organización del orden instituido en materia de sexualidad, el que para algunas autoras implicaba significaciones reproductoras de la desigualdad de género. Ellas mencionan, por ejemplo, la suposición de que hombres y mujeres tienen comportamientos diferenciados en materia de sexualidad porque son radicalmente distin- 
tos y opuestos entre sí; la regulación del sexo a través del amor; la pasividad erótica femenina; o el mito de la mujer-madre (Rubin, 1986; Fernández, 1992).

¿En qué medida esto ha evolucionado en las sociedades que podríamos llamar más «conservadoras»? ¿Cómo se han integrado las nuevas significaciones? ¿Cómo y con qué velocidad han operado estos cambios? ¿Qué papel viene a jugar la presencia del SIDA en este escenario de transformaciones? La emergencia de nuevos sentidos amenaza todo el orden social y sus repercusiones pueden ser incalculables. Sin embargo, las sociedades tienen establecidos ciertos mecanismos que permiten absorber estas significaciones emergentes, sin que por ello se desestabilice su organización básica.

Con respecto a esto Castoriadis señala que la institución de la sociedad comporta siempre defensas: las irrupciones del mundo en bruto serán siempre signos de alguna cosa, interpretadas y exorcizadas. El sueño y la enfermedad misma. El punto donde las defensas de la sociedad son más débiles es sin duda su propio imaginario instituyente, es también el punto sobre el cual ha sido inventada la defensa más fuerte: la denegación y el ocultamiento de la dimensión instituyente de la sociedad y la imputación del origen y del fundamento de la institución y de las significaciones que conlleva, a una fuente extra-social (Castoriadis, 1990).

En esta misma línea, Barthes (1958) postula la existencia de dos mecanismos principales que posibilitarían la absorción de las nuevas significaciones. Por una parte, la «recuperación», a través de la cual se refuerza el orden y las dinámicas ya existentes, y por otra, la «dilución», por la cual se priva al fenómeno de su sentido conflictivo integrándolo o anexándolo a la representación más tradicional o estereotipada. Es decir, se propone una coexistencia armónica de estos elementos o contenidos (Barthes, 1958, en Rivera, Rodó, Sharim y Silva, 1995, p. 9).

Rivera, Rodó, Sharim y Silva (1995), proponen, para el caso de la sociedad chilena, que en el terreno de la sexualidad, de lo instituyente propuesto en los años sesenta y reforzado por el movimiento feminista, se integró a lo instituido nuevos elementos, pero con ciertas limitaciones que lo entrampan en lo «antiguo». De esta forma, los cambios corresponden más a una ampliación de lo instituido, en que se invisibiliza y se niegan las contradicciones que ésta conlleva.
Tomando en cuenta todo lo anterior, ¿podríamos suponer que el SIDA, como un fenómeno relativamente reciente, vendría a ser un acelerador de las transformaciones sociales en materia de sexualidad y en la relación de poder entre hombres y mujeres?

Pensamos, solamente a modo de hipótesis, que esta enfermedad trae consigo una serie de nuevos significados que generan grandes contradicciones en las personas y que plantean limitaciones importantes en materia de sexualidad y de relación con el sexo opuesto. En una sociedad más bien tradicional en cuanto a las relaciones entre géneros, la amenaza de cambio en el orden instituido generaría, por el contrario, una reafirmación y un fortalecimiento de los modelos tradicionales de comportamientos sexuales asignados a cada género. El mundo «es así» y las relaciones entre hombres y mujeres serían naturalizadas. Es el «orden de las cosas»: hombres y mujeres somos diferentes y complementarios. Es la dominancia del momento imaginario sobre el racional-funcional (protección frente a la muerte).

Esto se reflejaría, por un lado, en la dificultad de hombres y mujeres para negociar conductas preventivas frente a la posibilidad de contagio, pero también sería observable a nivel de una parte del discurso público ${ }^{17}$ más bien «moralizador» $\mathrm{y}$ «negador» de la realidad de las relaciones entre hombres y mujeres en materia de sexualidad, adoptado por ciertos sectores de la sociedad.

La presencia de la enfermedad del SIDA, desencadenaría una «vuelta atrás» o por lo menos un «retardamiento» de los cambios. ¿Podríamos entonces pensar en una sociedad instituida resistiendo ante los «embistes» de nuevas y amenazantes significaciones instituyentes para el orden establecido, y de individuos adhiriendo inconscientemente a estas resistencias?

¿Cómo contribuir a la prevención del SIDA?

A modo de conclusión, pensamos que existen al menos dos niveles importantes de considerar cuando se habla de la prevención del SIDA. La llegada de esta epidemia podría ser pensada como un factor transformador social sólo si entendemos esta problemática desde un primer nivel más superficial, en el que la toma

17 Visible principalmente en algunas campañas públicas de prevención a través de ciertos medios de comunicación (pareja única, promoción de la abstinencia sexual, y sólo recientemente promoción del uso del preservativo). 
de conciencia del riesgo de contaminación y la amenaza de la muerte bastaría para una aceleración de los cambios en los modelos de sexualidad. Sin embargo, si consideramos un segundo nivel de análisis más profundo, podríamos hipotetizar que el SIDA vendría a ser más bien un factor retardador de las transformaciones sociales en cuanto a las desigualdades de poder entre géneros, principalmente por las resistencias inconscientes generadas ante la posibilidad de cambio.

Así, un primer nivel de intervención, importante pero no suficiente, tiene que ver con aprendizajes teóricos sobre la enfermedad y su forma de transmisión, así como con la promoción de capacidad de decidir sobre el propio cuerpo y del empoderamiento en la mujer. Pero como ya ha sido sugerido, no basta con esto, sobre todo porque una intervención de este tipo considera sólo un aprendizaje a nivel consciente y porque además se dirige sólo a la mujer como responsable de la prevención en la pareja.

En un segundo nivel, si se quiere poner en evidencia a través de la problemática del SIDA la desigualdad de géneros y las relaciones de poder entre hombres y mujeres, es entonces importante profundizar en el tema de la construcción social de estas desigualdades, expresadas en las relaciones de poder que se establecen entre ellos, y sobretodo, en la idea de la adhesión inconsciente a los modelos de género y su mantenimiento y reproducción a través de numerosas significaciones sociales imaginarias.

¿Cómo hacer esto? ¿A través de qué mecanismos podemos tratar de acceder a un nivel más profundo de análisis de tal forma de ayudar a la autonomía de las personas frente a la repetición de los modelos de comportamiento? En términos de Castoriadis, sólo a través de la promoción de un proceso reflexivo que contribuya a la creación de espacios de libertad que posibiliten el cambio social. Porque frente a la epidemia del SIDA no basta sólo con informar sobre los comportamientos necesarios para impedir la transmisión, sino que se hace necesaria una reflexión más profunda, de parte de los actores involucrados, sobre las determinaciones sociales que influencian su «imagen del mundo» (sobre las representaciones, pero también sobre los afectos y sobre las «intenciones» dentro y para los cuales la sociedad se instituye), sobre los comportamientos sexuales instituidos en una sociedad determinada y sobre las transformaciones sociales necesarias para evitar la propagación de la enfermedad.
¿Qué significa la promoción de la capacidad reflexiva y de la autonomía del ser humano?

Castoriadis señala a este respecto: «La autonomía del individuo se constituye cuando se establece otra relación entre la instancia reflexiva y las otras instancias psíquicas, como también entre su presente y su historia por medio de la cual él se ha hecho tal como es, permitiéndole escapar a la sumisión y a la repetición, de volverse hacia sí mismo, hacia las razones de sus pensamientos y los motivos de sus actos, guiado por la búsqueda de la verdad y la elucidación de su deseo (...) Ha cesado de ser el puro producto de su psiquis, de su historia, y de la institución que lo ha formado (...) la formación de una instancia reflexiva y deliberante, de la verdadera subjetividad, libera la imaginación radical del ser humano singular como fuente de creación y de alteración» (Castoriadis, 1990, p. 161).

Este mismo autor agrega: «La tradición significa que la legitimidad de la tradición no será cuestionada (...) la imaginación radical es reprimida y la dimensión instituyente de la sociedad denegada (...) el surgimiento de la interrogación ilimitada crea un eidos histórico nuevo (la reflexividad en sentido pleno, o la autorreflexividad) (...) La autonomía surge con la interrogación explícita no sobre los «hechos» sino que sobre las significaciones imaginarias y su fundamento $^{18}$, momento de creación, germen, porque la autonomía, tanto social como individual es un proyecto» (Castoriadis, 1990, p. 160).

¿Qué queremos subrayar con esta reflexión? Una vez más, que para intentar combatir la epidemia del SIDA no basta sólo con señalar la existencia de una relación de dominación entre hombres y mujeres, sino que se hace también necesario interrogarse y reflexionar sobre la permanencia de este sistema de poder, sobre la manera en la que éste se expresa en el proceso de negociación sexual y sobre las significaciones imaginarias sociales que subyacen a él.

Evidentemente, llegaremos nuevamente a la cuestión de los modos para acceder a las significaciones imaginarias sociales. Para intentar comprender el imaginario de una sociedad el investigador debe interpretar las representaciones (la mayor parte del tiempo solamente a través de la palabra), y las prácticas de los individuos. Pero esto será siempre una "interpretación

18 Subrayado agregado. 
de una interpretación del mundo"... ¿Es posible hacerlo de otro modo? Pregunta difícil de responder, ya que éste ha sido uno de los temas claves en la discusión epistemológica hasta nuestros días. Castoriadis dice: “... en el caso del imaginario, el significado al que lleva el significante es casi inalcanzable como tal, y por definición su "modo de ser" es un modo de "no ser"... ¿Cómo alcanzar esta a-realidad última? Ella no se muestra como "las cosas en sí", sino que a partir de sus consecuencias, de sus resultados, de sus derivados (Castoriadis, 1975, p. 214).

Si pensamos en los programas de prevención que tienen como principal objetivo el obtener un cambio de las prácticas sexuales "en los tiempos del SIDA", un trabajo de reflexión personal más profunda (individual o grupal) podría tal vez ser más eficaz que el solo aprendizaje teórico.

Para finalizar, citaremos a Bourdieu (1998): «esta constatación de la constante transhistórica de la relación de dominación masculina (...) condena en efecto a hacerse la pregunta, siempre ignorada, sobre el trabajo histórico recomenzado una y otra vez y que es necesario para arrancar la dominación masculina a la historia, y sobre los mecanismos y las acciones históricas que son responsables de su aparente deshistorización, y que toda política de transformación histórica debe conocer, para no arriesgar el autocondenarse a la impotencia» (Bourdieu, 1998, p. 110).

\section{Referencias}

Agenda de Salud. Publicación trimestral del Programa Mujeres y Salud de ISIS Internacional. Santiago: ISIS Internacional.

Barthes, R. 1958. Mythologies. París : Seuil.

Bourdieu, P. 1998. La domination masculine. París: Editions du Seuil.

Castoriadis, C. 1975. L'institution imaginaire de la société. París: Editions du Seuil.

Castoriadis, C. 1990. Le Monde Morcelé. Les Carrefours du Labyrinthe - 3. París : Editions du Seuil.

Coria, C. 1994. Negociación y género: mujeres que ceden para no negociar. Ponencia para el Congreso Latinoamericano de psicoterapia analítica de grupos, Buenos Aires.

Dayan-Herzbrun, S. (s/d). Notes du séminaire «Rapports de genre au Maghreb et au Machreb» EHESS, 2000-2001.

De Barbieri, T. 1992. Sobre la categoría de género: una introducción teórico-metodológica. En R. Rodríguez (ed). Fin de siglo y cambio civilizatorio. Ediciones de las $\mathrm{Mu}-$ jeres $n^{\circ} 17$. Santiago: ISIS Internacional.

Delphy, Christine. 1988. L'ennemi principal. Economie politique du patriarcat. Paris: Syllepse.

Fernández, A.M. (comp.). 1992. Mujeres en la imaginación colectiva. Una historia de discriminación y resistencias. Buenos Aires: Paidós.

Foucault, M. 1976. Histoire de la sexualité I. La Volonté de savoir. París: Gallimard.

Rivera, D., Rodó, A., Sharim, D., Silva, U. (1995). Relaciones de género y sexualidad. Informe de Investigación. Santiago: SUR Profesionales. Documento de trabajo $\mathrm{n}^{\circ} 153$.

Rubin, G. 1986. El tráfico de mujeres: notas sobre la economía política del sexo. Nueva Antropología (México), $n^{\circ} 30$.

Takeuti, N. (s/d). Imaginario social "mortifero": A questao da delinqüência juvenil no Brasil. Documento del Curso dictado en el contexto del DEA Sociologie du Pouvoir, Laboratoire de Changement Social, París 7, noviembre, 2000.

Vidal, L. 2000. Femmes en temps de SIDA: Expériences d'Afrique. París: PUF. 